\title{
PanDao fabrication cost impact analysis software tool for optical designers
}

\author{
Marco Tinner ${ }^{\mathrm{a}}$, Irina Livshits ${ }^{\mathrm{b}}$ and Oliver Faehnle ${ }^{\mathrm{a}}$ \\ a PanDao GmbH, St. Gallen, Switzerland, \\ ${ }^{\mathrm{b}} \mathrm{CAD}$ for Opto-Information \& energy saving systems", ITMO University, St. Petersburg, Russia
}

\begin{abstract}
A new software tool, called PanDao, for optical system designers is presented enabling during the design stage a simulation of the optimum fabrication chain and technologies needed as well as a fabrication cost impact analysis of design parameters and tolerances.
\end{abstract}

During the generation of optical systems, three different entities are involved subsequently:

(a) initially, optical system designers are translating performance parameters into optical system parameters such as types of glass used, lens geometries, surface shape accuracies, roughness and mid-spatial frequency errors as well as types of coatings applied.

(b) subsequently, optics fabrication designers translate optical systems' parameters into a well-designed fabrication chain employing machinery with optimized sets of fabrication parameters such as abrasives being used, machining kinematics, resonance frequencies, or sputtering rates being applied.

(c) finally, production managers are using the installed optical fabrication chain to manufacture the optical system applying optimized batch sizes and well-trained operators at high automatization levels.

Traditionally, it's the optical designers who are negotiating with customers about the product "optical system" including specifications, prices and issues concerning fabrication, coating, mounting and delivery of product.

While optical designers are well supported by software tools helping them to design the optimum set of optical elements, there are no such tools existing to design the optimum fabrication chain needed for production. As far as optical fabrication is concerned, optical designer's decisions are restricted to their personal experiences from earlier negotiations with their company's or their suppliers optics workshop. This is because optical fabrication technologies are not part of optical designer's training; patricularly chemical engineering, material science, machine tool metrology, mechanical engineering, abrasive machining, fabrication process' parameters control and a profound understanding on the science behind the "golden hands of the opticians".

Recently, within the PanDao software project ${ }^{1}$, such a tool has been developed. Based on the know how gained from decades of academic and industrial State-of-the-Art manufacturing and experiences, the PanDao expert system has been developed. The PanDao software has been exposed to stress testing within both industrial production environments and within optical workshops located in research institutes for the generation of high end optical systems for space, medical an defense applications.

Reading in lens parameters and tolerances as generated by commercially available optical design software tools and, additionally, considering commercial order's informations such as "total number of lenses to be produced" and "batch sizes to be delivered", PanDao takes all known optical fabrication techniques (such as SPDT ${ }^{2}$, bonnet polishing ${ }^{3}, \mathrm{IBF}^{4}, \mathrm{FJP}^{5}$ ) into account to generate informations about fabrication during the design stage. That way, optical designers can test their optics designs for producibility and the essential optical fabrication technologies needed for production. Furthermore, fabrication cost-impact analyses of optical design parameters are enabled based on which design parameters can be optimized for minimum fabrication cost. Thereforth, PanDao describes during the optical design process the whole fabrication chain including fabrication cost, coating cost, testing cost and centering cost.

In the following, PanDao's fabrication cost and technology analysis of a plano-aspherical lens (see Fig.1) is presented, featuring $220 \mathrm{~mm}$ diameter to be produced in 500 pieces quantity.

PanDao (Fig.2) identifies sub-aperture cnc grinding with a final ccp-bonnet polishing to be the optimum fabrication chain to be applied. Total fabrication cost per lens is about $185 €$ (taking an average sized central-European company into account: without companys overhead cost and material cost); total orders fabrication cost is 92 ' 500 $€$. In the following, three examples are given, demonstrating how optical design adjustments can impact on fabrication cost. 
- Changing lens diameter from $220 \mathrm{~mm}$ to $210 \mathrm{~mm}$ (and filling in the space by e.g. a metal ring), brings cost per lens down to $176.8 €$ resulting in $4.4 \%$ (or 4'100 €) fabrication cost reduction overall (Fig.3).

- Changing lens' asphericity (maximum distance from best removal spherical surface to aspherical surface to be generated) from $4 \mu \mathrm{m}$ down to $2 \mu \mathrm{m}$ reduces fabrication cost per lens down to $155.5 €$ per lens resulting in $15.9 \%$ (or 14'750 €) fabrication cost reduction overall (Fig.4). In this case, it is cheaper to grind and polish the removal spherical surface first and to use aspherics polishing techniques subsequently, rather than to grind and polish the aspherical surface directly.

- Finally, introducing more stringent mid-spatial tolerancing, e.g. reducing the smallest allowed MSW (mid-spatial wavelength) from $5 \mathrm{~mm}$ down to $0,2 \mathrm{~mm}$ leads to an increase in fabrication cost of $16.6 \%$ (or 15’355 €) because a final IBF shaping step is needed (Fig.5).

In conclusion, a new optical design software tool, called PanDao, has been developed enabling the design of the optimum fabrication chain depending on optical design parameters and tolerances. That way, fabrication cost can be minimized already in the design stage of optical systems.

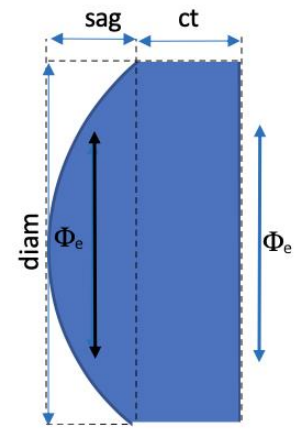

SIDE 1
clearAperture $\Phi$ e $200 \mathrm{~mm}$
AR coating
$3 / 3(2)$
$4 / 1^{\prime}$
$5 / 3^{*} 0.064$
Sq roughness: $1 \mathrm{~nm}$ rms
sag $40 \mathrm{~mm}$
smallest mitSpatialWavelength $5 \mathrm{~mm}$
radius of removal sphere $660 \mathrm{~mm}$
asphericity 4 mm
smalles radius of curvature $673 \mathrm{~mm}$
contains concave parts? no

\begin{tabular}{|c|c|}
\hline PANDAO & \multirow{9}{*}{$\begin{array}{l}\text { SIDE } 1 \\
\text { clearAperture } \Phi \text { e } 206 \mathrm{~mm} \\
\text { AR coating } \\
3 / 1(0.2) \\
4 /- \\
5 / 3^{*} 0.063 \\
\text { Sq roughness: } 1.5 \mathrm{~nm} \mathrm{rms} \\
\text { sag } 0 \mathrm{~mm}\end{array}$} \\
\hline glass: BK7 & \\
\hline DIMENSIONS & \\
\hline center thickness ct $60 \mathrm{~mm}$ & \\
\hline \multirow{2}{*}{$\begin{array}{l}\text { ct tolerance } 0.05 \mathrm{~mm} \\
\text { diameter } 220 \mathrm{~mm}\end{array}$} & \\
\hline & \\
\hline PRODUCTION ORDER & \\
\hline totalNumber pcs 500 & \\
\hline batchSize pcs: $\quad 50$ & \\
\hline
\end{tabular}

Fig.1. Plano-aspherical BK7 lens with 500 pieces to be produced: to be analized by PanDao software tool

\begin{tabular}{|llll|}
\hline \multirow{2}{*}{ Side 1: } & Stage 1: & Stage 2: & \multicolumn{1}{c|}{ Stage 3: } \\
& cnc sub aperture rough & cnc sub aperture grindingccp bonnet \\
& grinding & fabrication price 29.77€ & fabrication price $99.19 €$ \\
Side 2: & fabrication price 11.79€ & & \\
& curve generator rough & cnc full aperture flats & cnc full aperture flats \\
& grinding & grinding & polishing \\
& fabrication price 9.29€ & fabrication price 17.62€ & fabrication price 17.41€ \\
Total fabrication cost for this lens: 185.08€. & & \\
Investbudget for installing machinery for this fab-chain: 770000€ & \\
\hline
\end{tabular}

Fig.2. PanDao analysis of the plano-aspherical BK7 lens (Fig.1) with per lens $185 €$ fabrication cost excluding $8.7 €$ center grinding cost, $18.5 €$ coating cost and $18.2 €$ testing cost. 


\begin{tabular}{|llll|}
\hline Side 1: & $\begin{array}{l}\text { Stage 1: } \\
\text { cnc sub aperture }\end{array}$ & $\begin{array}{l}\text { Stage 2: } \\
\text { cnc sub aperture }\end{array}$ & $\begin{array}{l}\text { Stage 3: } \\
\text { ccp bonnet }\end{array}$ \\
& rough grinding & grinding & fabrication price \\
& fabrication price & fabrication price & $94.71 €$ \\
Side 2: & $11.28 €$ & $28.43 €$ & \\
& curve generator & cnc full aperture flats cnc full aperture flats \\
& rough grinding & grinding & polishing \\
& fabrication price & fabrication price & fabrication price \\
& $8.89 €$ & $16.84 €$ & $16.63 €$ \\
Total fabrication cost for this lens: $176.79 €$. & \\
Investbudget for installing machinery for this fab-chain: $770000 €$
\end{tabular}

Fig.3. PanDao analysis of the plano-aspherical BK7 lens (Fig.1): reducing lens diameter from $220 \mathrm{~mm}$ down to $210 \mathrm{~mm}$ gains $4.4 \%$ total fabrication cost reduction.

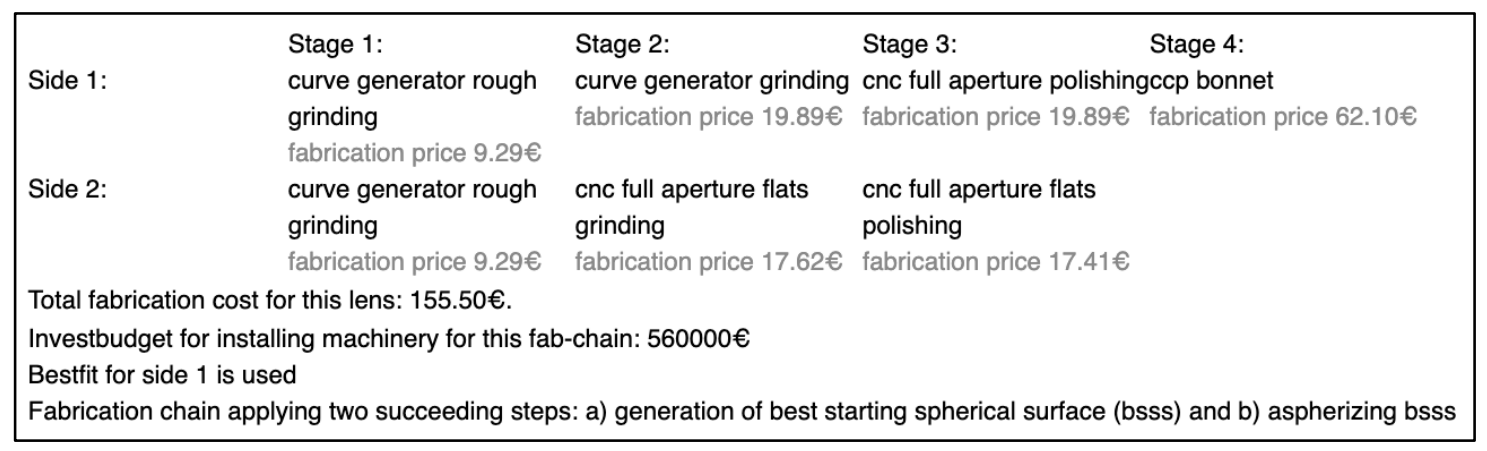

Fig.4. PanDao analysis of the plano-aspherical BK7 lens (Fig.1): reducing asphericity from $4 \mu \mathrm{m}$ down to $2 \mu \mathrm{m}$ gains $15.9 \%$ total fabrication cost reduction.

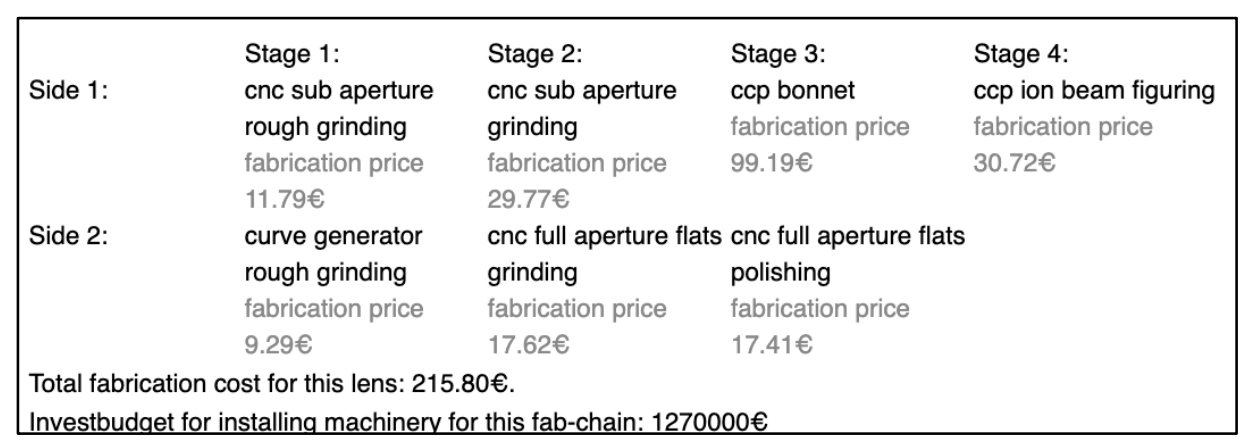

Fig.5. PanDao analysis of the plano-aspherical BK7 lens (Fig.1): reducing mid-spatial wavelength from $5 \mathrm{~mm}$ down to $0,2 \mathrm{~mm}$ generates $16.6 \%$ more fabrication cost.

\section{References}

1. Webside: https://www.PanDao.ch

2. Jiwang Yan, J. Tamaki, K. Syoji, T. Kuriyagawa, "Single-point diamond turning of CaF2 for nanometric surface", The International Journal of Advanced Manufacturing Technology, Volume 24, Issue 9, pp 640646, 2004

3. David Walker; A. T. Beaucamp ; David Brooks ; Richard Freeman ; Andrew King ; Gerry McCavana ; Roger Morton ; David Riley ; John Simms; Novel CNC polishing process for control of form and texture on aspheric surfaces. Proc. SPIE 4767, Current Developments in Lens Design and Optical Engineering III, 2002;

4. Franz Thomas, and Thomas Hänsel, "Ion beam figuring (IBF) solutions for the correction of surface errors of small high performance optics." Optical Fabrication and Testing. Optical Society of America, 2008;

5. Oliver Faehnle, H. van Brug and H. Frankena, "Fluid Jet Polishing of optical surfaces", Applied Optics 37(28), 6771-6773, 1998; 